\title{
Genetic and environmental influences on drug use and abuse/dependence in male and female twins
}

\author{
Marianne B.M. van den Bree ${ }^{\mathrm{a}, *}$, Eric O. Johnson ${ }^{\mathrm{b}}$, Michael C. Neale ${ }^{\mathrm{c}}$, Roy W. Pickens ${ }^{\mathrm{a}}$ \\ ${ }^{a}$ Intramural Research Program, National Institute on Drug Abuse, Addiction Research Center, P.O. Box 5180, Baltimore, MD 21224, USA \\ ${ }^{\mathrm{b}}$ Department of Psychiatry, Henry Ford Health Science Center, Detroit, MI 48202, USA \\ ${ }^{\mathrm{c}}$ Department of Psychiatry, Medical College of Virginia, Virginia Commonwealth University, Richmond, VA 23298, USA
}

Received 16 January 1998; accepted 15 May 1998

\begin{abstract}
Twins were recruited through alcohol and drug treatment programs. With structural equation modeling, genetic and environmental estimates were obtained for use and DSM-III abuse/dependence of sedatives, opioids, cocaine, stimulants, and cannabis as well as any illicit drug. Analyses were conducted separately for males and females. Models included thresholds based on population prevalence of use or abuse/dependence and ever having been in treatment. Genetic influences were found for most measures. They were generally stronger for males than females and for clinical diagnoses of abuse/dependence compared to use. Common environmental influences played a greater role in use than abuse/dependence. (C) 1998 Elsevier Science Ireland Ltd. All rights reserved.
\end{abstract}

Keywords: Drug use; Drug abuse/dependence; Twins; Genetic and environmental influences; Gender

\section{Introduction}

Several twin studies suggest both genetic and environmental influences are involved in illicit drug use/dependence (Pedersen, 1981; Grove et al., 1990; Pickens et al., 1991; Gynther et al., 1995; Jang et al., 1995; Tsuang et al., 1996). However, because the low prevalence and illicit nature of drug abuse make subject recruitment and data collection difficult, most of these studies have collapsed subjects across drug class or gender, although epidemiological, clinical, and animal studies have shown drug abuse liability to vary by both variables (Regier et al., 1990; Kosten et al., 1993; Morishima and Whittington, 1995; Warner et al., 1995; Caetano and Schafer, 1996; Koob and Nestler, 1997).

To date, only two twin studies have reported genetic influences on specific classes of illicit drugs. In one

\footnotetext{
* Corresponding author. Tel.: + 1410 5501634; fax: + 1410 5501202; e-mail: mvandenb@intra.nida.nih.gov
}

study, prescription medication use ('tranquilizers' and 'sleeping pills') was analyzed for genetic influence. While these drug classes are pharmacologically similar, some evidence for drug specificity was reported. A heritability estimate of 0.28 was found for tranquilizers but no evidence of heritable influences was found for sleeping pills (Pedersen, 1981). In another study, heritability estimates were obtained for various classes of illicit drugs in a large sample of male twins who both served in the military between 1965 and 1975 . Heritability estimates ranged from 0.25 for $\mathrm{PCP} /$ psychedelics to 0.44 for stimulants (Tsuang et al., 1996).

Only one twin study has reported heritability estimates for drug abuse in males and females separately. This study was based on a treatment-based sample of twins where probands also were comorbid for alcohol abuse/dependence. Heritability of DSM-III drug abuse/ dependence was found to be 0.31 for males and 0.22 for females (Pickens et al., 1991). Most studies have been based on only a single gender (Tsuang et al., 1996) or 
combined results for males and females (Grove et al., 1990; Gynther et al., 1995; Jang et al., 1995). To date, no study has examined heritability of drug abuse by both drug class and gender of subject.

In the present study, magnitude of genetic and environmental influence was estimated on lifetime use and clinical diagnosis of drug abuse/dependence. Probands were twin pair members ascertained from individuals enrolled in alcohol/drug treatment programs. Estimates of genetic, common environmental, and specific environmental influences were obtained after adjusting for prevalence of drug use or abuse/ dependence as well as treatment status in the general population using thresholds based on population estimates from the Epidemiological Catchment Area (ECA) survey (Eaton and Kessler, 1985). In addition, models adjusted for the occasional double ascertainment of probands. Heritability estimates were obtained for illicit drug use as well as the more severe clinical measure of abuse/dependence. Analyses were conducted separately for the specific drug classes (sedatives, opioids, cocaine, stimulants, cannabis), as well as for any drug use and abuse/dependence, and also separately for male and female twins, allowing estimation of gender-specific genetic and environmental influences.

\section{Method}

\subsection{Sample}

For years 1982-1988, clients in 16 public and private alcohol and drug abuse treatment and follow-up programs in Minnesota were screened for twin status. If they were a twin and willing to participate, their co-twin was also contacted. Of a total sample of 392 twin pairs, a convenience subsample of 188 twin pairs (56 monozygotic male (MZM), 66 dizygotic male (DZM), 38 monozygotic female (MZF), and 28 dizygotic female (DZF) pairs) were interviewed to assess drug and alcohol use and dependence and related psychiatric conditions. Opposite-sex DZ twin pairs did not participate in the interview phase of the study, and therefore were not included in the present analyses. Thirty-five percent of the sample was female and $92 \%$ was Caucasian, the mean age was 35.4 years (range 15-63), and $35 \%$ of probands and $58 \%$ of co-twins were married or cohabitating. The sample differs from an earlier report (Pickens et al., 1991), which included only probands meeting DSM-III criteria for alcohol abuse and/or dependence. Complete details of subject ascertainment, zygosity determination, and other sample characteristics have been published elsewhere (Pickens et al., 1991).

\subsection{Genetic theory}

Similarities between biological relatives may be due to either genetic factors or environmental influences shared with other family members (including parental rearing environment, friends, but also intrauterine environment). Environmental effects not shared by family members tend to make family members less alike. MZ twins are genetically identical, and, if reared together, also have family environment in common. DZ twins raised together also share family environment, but have, on average, only half of their genes in common. Under the assumptions of equal environmental similarity for both twin types, random mating, and absence of gene-environment interaction or correlation, greater similarity of $\mathrm{MZ}$ than $\mathrm{DZ}$ twins suggests additive genetic influences. A MZ correlation of less than 1.0 suggests specific environmental influences. A DZ correlation approximately half that for $M Z$ twins suggests additive genetic influences, while a DZ correlations greater than half the $\mathrm{MZ}$ correlation suggests common environmental influences. A DZ correlation less than half the MZ correlation suggests non-additive genetic influences. The principles of twin methodology are outlined in detail in several sources (Heath et al., 1989; Neale and Cardon, 1992).

\subsection{Analysis}

Data on illicit drug use ( $>5$ times in lifetime) and DSM-III diagnoses of drug abuse and/or dependence were obtained from information collected by the Diagnostic Interview Schedule, Version III (DIS-III) (Robins et al., 1981) using standard algorithms (Boyd et al., 1985). Information on specific drugs was combined into five drug categories: sedatives (includes barbiturates and tranquilizers); opioids (includes heroin and other opiates); stimulants (amphetamines); cannabis (marijuana); and cocaine. Because hallucinogen use was very infrequent, it was not included in the analyses. New variables 'any drug use', 'any drug dependence' and 'any drug abuse/dependence' were created by combining information on specific drug categories.

In the DSM-III diagnostic system, both abuse and dependence diagnoses could be obtained for cannabis, sedatives, opioids, and stimulants, while for cocaine only the diagnosis of abuse is supported. A DSM-III diagnosis of abuse required evidence of drug-induced interference with social or occupational functioning and evidence of pathological pattern of use. Diagnosis of dependence required evidence of tolerance and/or withdrawal symptoms, with cannabis dependence also requiring evidence of impairment in social or occupational functioning (American Psychiatric Association, 1980). 
For genetic analysis of the dichotomous data (drug use, abuse/dependence), tetrachoric or liability correlation coefficients were calculated (Neale and Cardon, 1992). Liability to drug abuse/ dependence is an estimate of the magnitude of prevalence of the diagnosis among co-twins of affected probands compared to prevalence in the general population (Reich et al., 1972). Under the influence of multiple genes in combination with multiple environmental factors, liability is normally distributed. A threshold, determined by the population prevalence for drug abuse/dependence, represents the cut-off between unaffected and affected individuals. Individuals with scores above the threshold will receive a diagnosis of drug abuse/dependence. Similarly, individuals with scores above the threshold for drug use liability will try drugs.

Population prevalence rates of drug use, diagnoses of abuse/dependence as well as treatment status were calculated from data provided by the Epidemiological Catchment Area (ECA) study (Eaton and Kessler, 1985). The ECA study employed the same DIS-III interview that was used in the present study. Prevalence rates were poststratified to adjust for age, sex, and racial characteristics of the twin sample. A weighting function (SW1) was used to eliminate biases in the ECA dataset due to oversampling of certain segments of the population. Since the ECA was a longitudinal study, two waves of data were publicly available at the time of the present analysis. In case of negative or missing diagnoses in wave 1 , wave 2 information for the same subject was substituted.

For genetic analysis, structural equation models were specified in the statistical program Mx (Neale, 1997). Input were tables of raw data. For each analysis, population prevalence rates, as obtained from the ECA survey, were used by Mx to estimate the threshold for use, or abuse/dependence of a specific drug type. In addition to a threshold for use or abuse/dependence of a drug, a threshold for ascertainment through alcohol/ drug clinics was estimated to account for treatment status of the twin sample. ECA prevalence rates were entered in separate groups in $\mathrm{Mx}$ in order to estimate the thresholds jointly (bivariate analysis) with the genetic and environmental parameters of the model. All analyses were conducted separately for males and females, with gender-specific threshold estimates for drug use or abuse/dependence, as well as treatment status.

Models allowed for the specification of doubly ascertained twin pairs. For both MZ and DZ twins, input data consisted of eight of the 16 possible cells of the $2 \times 2 \times 2 \times 2$ contingency tables crosstabulating twin 1 and twin 2 ascertainment and diagnostic status. Only eight cells per group were included because probands were necessarily ascertained.

Relative contributions of latent factors were estimated in a model including additive genetic influences
(A), as well as common (C), and specific environmental (E) factors (ACE model). Relative contributions of genetic influences $\left(h^{2}\right.$, or heritability estimate) as well as common and specific environmental influences $\left(c^{2}\right.$ and $e^{2}$, respectively), were obtained by dividing their variation by the total phenotypic variation (genetic and environmental influences combined).

The program fitted models by a minimum chisquared method, which is generally superior to maximum likelihood methods, when cell frequencies are low (Agresti, 1990). Fit of the model was evaluated with the probability $(P)$ value of the associated $\chi^{2}$ statistic, with seven degrees of freedom (df). A small $P$-value suggests considerable discrepancy between model and observed data, while larger values represent increased correspondence, indicating a better fit. Another indicator is Akaike's Information Criterion (AIC) (calculated as $\chi^{2}-2 *(\mathrm{df})$ ) (Akaike, 1987). In comparing models, low AIC values indicate a more parsimonious explanation of the data. The fit of (nested) models excluding either $\mathrm{A}, \mathrm{C}$, or both, was compared against the full ACE model to determine the significance of these individual parameters. In addition, confidence intervals (95\%) around heritable and environmental estimates were requested in $\mathrm{Mx}$ to evaluate parameter significance.

\section{Results}

Table 1 gives percentages of illicit drug use (five times or more) and diagnoses of DSM-III drug abuse/dependence in lifetime for male and female probands and co-twins separately. Prevalence of use and abuse/dependence of each drug class was consistently higher for probands than co-twins. Compared to male probands, female probands had higher prevalence rates of repeated use of one drug type (sedatives), and most drug diagnoses (with the exception of stimulant and cannabis abuse and/or dependence, and stimulant dependence). Prevalence rates for any repeated use as well as any diagnosis of drug abuse/dependence were also higher for female probands. However, among co-twins use and abuse/dependence of specific drug types were more prevalent for males than females (with the exception of opiate use, and sedative and opiate abuse/dependence). Male co-twins also had higher use and abuse/dependence of any drug. Use of any drug was reported by $65-71 \%$ of probands and $39-46 \%$ of co-twins. A diagnosis of abuse and/or dependence was found in approximately $48-56 \%$ of probands and $18-28 \%$ of co-twins, while a diagnosis of dependence occurred less frequently $(34-47 \%$ for probands and $12-21 \%$ for cotwins). Both use and abuse/dependence were more prevalent for cannabis than for most other drugs (although similar prevalence levels were obtained for cannabis and stimulant dependence for male co-twins, and 


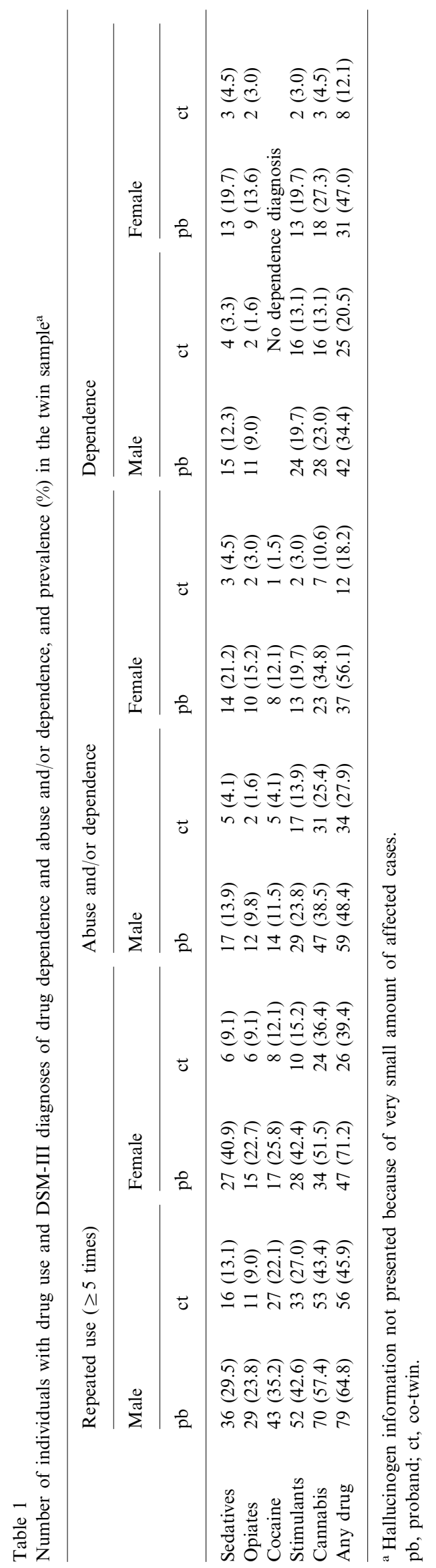


Table 2

Numbers of discordant (DC) and concordant (CC) affected twin pairs as well as tetrachoric correlation coefficients ( $\rho$ ) for drug use and DSM-III diagnoses of drug dependence and abuse and/or dependence

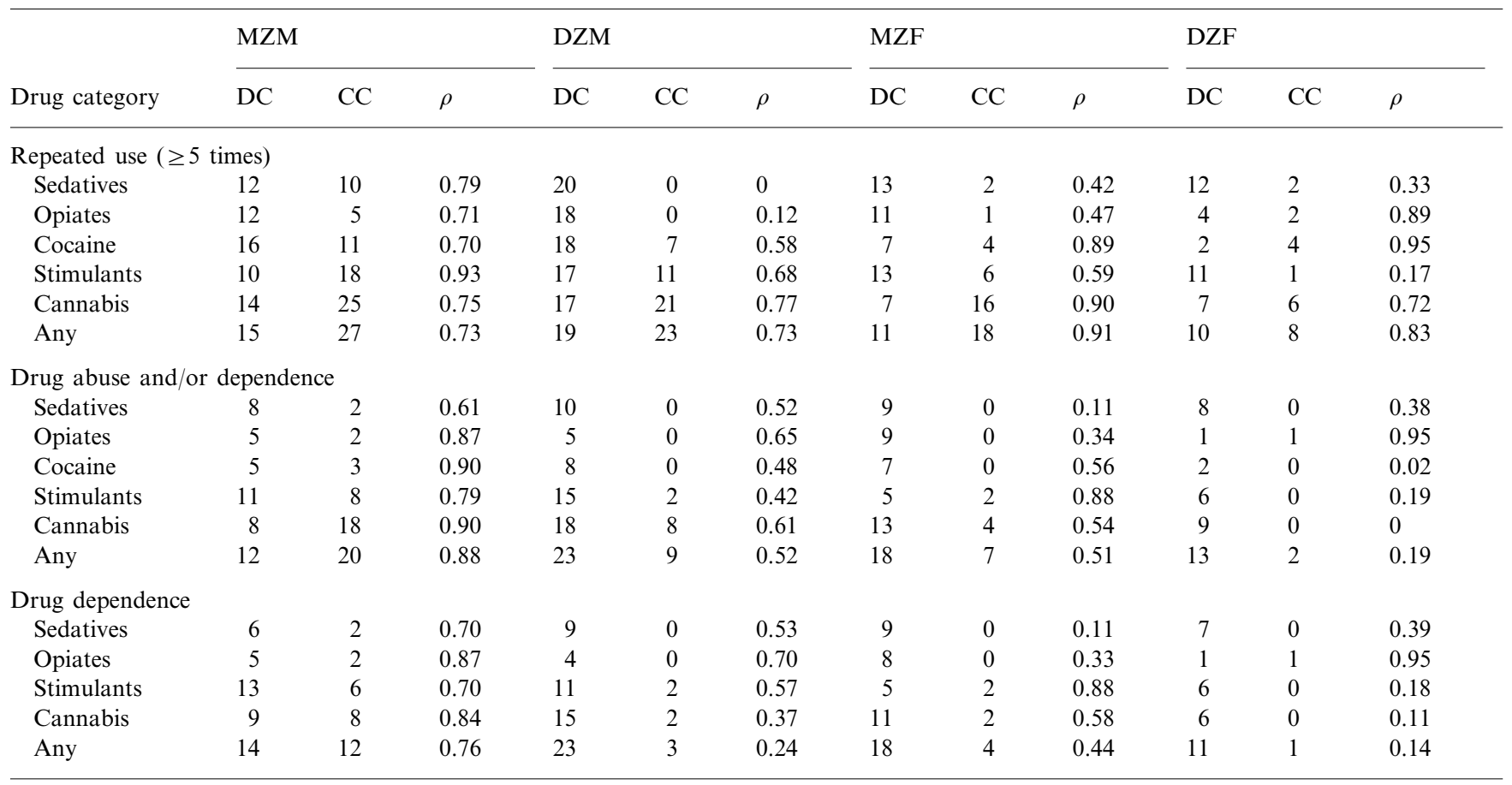

MZM, monozygotic males; DZM, dizygotic males; MZF, monozygotic females; DZF, dizygotic females.

for cannabis and sedative dependence for female cotwins). Use and abuse/dependence of opiates was low compared to other drug classes, whereas for females prevalence of sedative use of co-twins, cocaine abuse and/or dependence of both probands and co-twins, and stimulant dependence of co-twins was also relatively low.

Number of discordant and concordant affected twin pairs, as well as tetrachoric correlation coefficients for the measures under study are given in Table 2 for the four twin groups separately. Repeated use, drug abuse and/ or dependence, and drug dependence formed a hierarchy with increasing severity and decreasing numbers of pairs with at least one affected individual. Tetrachoric correlation coefficients were larger for measures with greater numbers of concordant affected, relative to discordant (and concordant unaffected) twin pairs. Prevalence in the general population also influenced the correlation coefficients. Given a certain number of concordant affected versus other twin pairs, lower correlations were obtained for measures with a relatively high prevalence in the general population, than for more rare ones.

Comparison of $\mathrm{MZ}$ and $\mathrm{DZ}$ tetrachoric correlation coefficients suggested either additive genetic and/or common environmental influences contributed to all classes of drug use and abuse/dependence, for both males and females. For some variables, DZ correlation coefficients that approached or exceeded those for MZ twins suggested limited evidence for additive genetic influences (for males: cannabis and any drug use; for females: opiate and cocaine use; sedative and opiate abuse/dependence). For some other variables DZ correlation coefficients were considerably lower than those for MZ twins, suggesting a limited role for common environmental influences (for males: sedatives and opiate use; for females: cocaine abuse and/or dependence; stimulant and cannabis abuse/dependence).

Fig. 1 presents results from structural equation modeling of drug use. For males, heritable influences were estimated for all drug classes, but relatively small additive genetic contributions were found for cannabis and any drug use. Over $50 \%$ of total phenotypic variation was explained by additive genetic factors for sedative use, opiate use, and stimulant use. No common environmental influences were found for opiate use, while they were very small for sedative use. For the other classes of drug use, estimated common environmental factors ranged from 0.33 (cocaine) to 0.62 (cannabis). A relatively large common environmental factor was also estimated for any drug use (0.61).

For females, influence of additive genetic factors was considerable for stimulant and cannabis use ( $>50 \%$ of explained variation), while a smaller genetic effect was estimated for sedative use. Genetic factors explained $23 \%$ of variation in any drug use for females. For two classes of drug use (opiates and cocaine), no genetic influences were detected. Common environmental ef- 

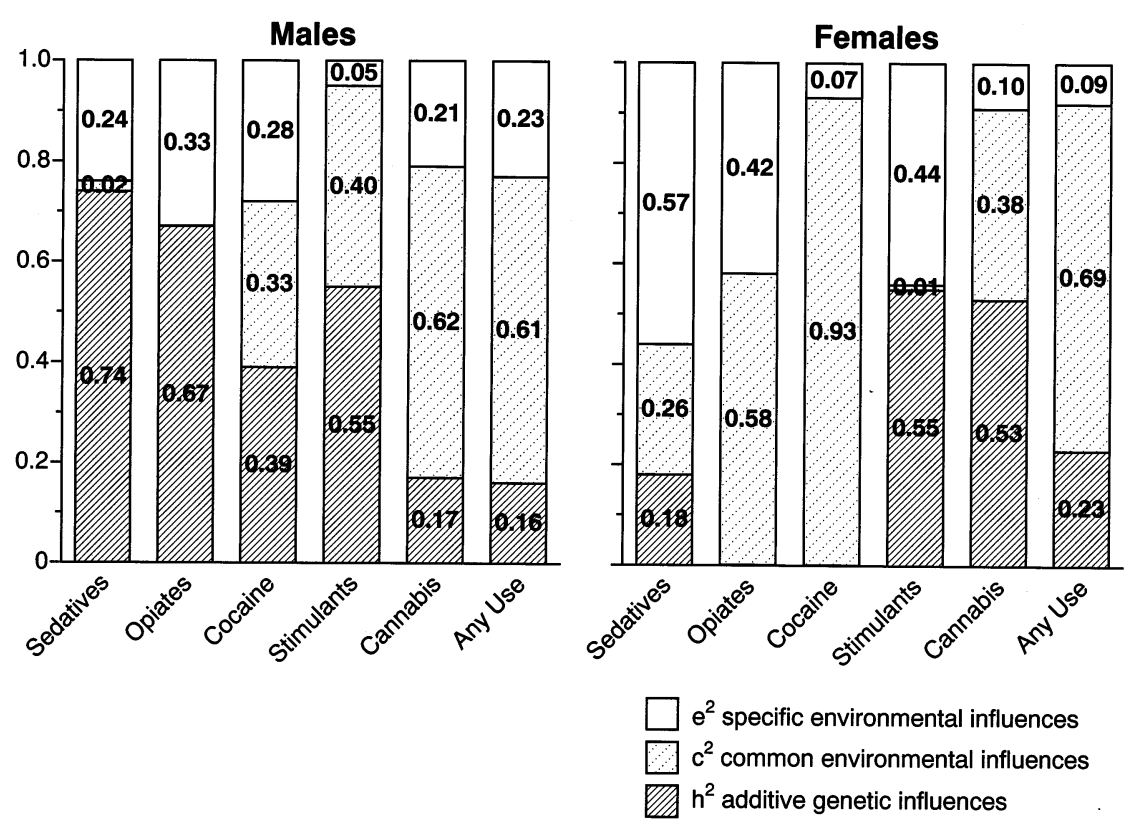

Fig. 1. Additive genetic, common environmental, and specific environmental influences on illicit drug use for males and females.

fects were found for all drug use classes, including any drug use, with magnitudes ranging from almost zero (stimulants) to greater than 0.9 (cocaine).

Evidence of genetic influences on DSM-III drug abuse and/or dependence in males was found for all drug classes, including any drug abuse and/or dependence (Fig. 2). All heritability estimates exceeded 0.5, indicating that genetic influences were more important than the two environmental factors combined. No evidence of common environmental influences was found for sedative and stimulant abuse and/or dependence, while estimates for the other classes ranged between 0.18 and 0.30 . A common environmental factor of 0.09 was estimated for any drug abuse/dependence.

For females, evidence of genetic influences was found for cocaine, stimulants and cannabis abuse and/or dependence, with heritability estimates ranging from 0.42 (cocaine) to 0.73 (stimulants). A genetic influence of 0.47 was estimated for any drug abuse and/or dependence. No heritable component was found for abuse and/or dependence of sedatives, and opiates. The effects of common environmental factors were non-zero for all classes of drug abuse and/or dependence, except for cannabis. They were relatively large for sedative $(0.29)$ and opiate (0.60) abuse and/or dependence, but limited in size for the other drug classes. For any drug abuse and/or dependence, only $4 \%$ of variation was estimated to be of common environmental origin.

Fig. 3 presents estimates of genetic and environmental contributions to the more specific case of DSM-III drug dependence. For males, heritable influences were found for all drug categories, as well as for any drug dependence, with explained variance attributable to genetic factors ranging from 0.48 (opiates) to 0.78 (cannabis). Evidence of common environmental influences was limited for all classes but one $(0.40$ for opiates). No evidence of common environmental influences on any drug dependence was found.

For females, heritable influences were estimated for stimulants, cannabis and any drug dependence, while no evidence for genetics effects was found for sedative and opiate dependence. Common environmental factors were found for all categories of drug dependence, but tended to be small, except for sedative (0.30) and opiate dependence (0.62). Eleven percent of variation in any drug dependence was explained by common environmental influences.

For all structural equation models with illicit drug use and drug abuse/dependence, $P$-values greater than 0.05 in combination with negative AIC values indicated consistency between model and data. The only exception was the model for cocaine abuse and/or dependence for males, which yielded a $\chi_{(7)}^{2}$ of $40.08, P<0.05$ $(\mathrm{AIC}=26.08)$. Excluding this model, $\chi^{2}$ values ranged from $10.99(P=0.14$, AIC $=-3.01)$ for sedative use to $3.16(P=0.87, \mathrm{AIC}=-10.84)$ for opiate abuse and $/$ or dependence in males, and $11.50(P=0.12, \mathrm{AIC}=-$ 2.5) for cocaine abuse and/or dependence to $1.82(P=$ $0.97, \quad$ AIC $=-12.18$ ) for stimulant abuse and/or dependence for females.

Confidence intervals (95\%) around genetic and common environmental estimates were wide, the difference between upper and lower limits being larger than 0.5 for almost all analyses (data available from first author upon request). For specific environmental influences, confidence intervals were less wide (the smallest interval, for cocaine abuse and/ or dependence in males had a lower limit of 0.05 and a upper limit of 0.15 , while the 

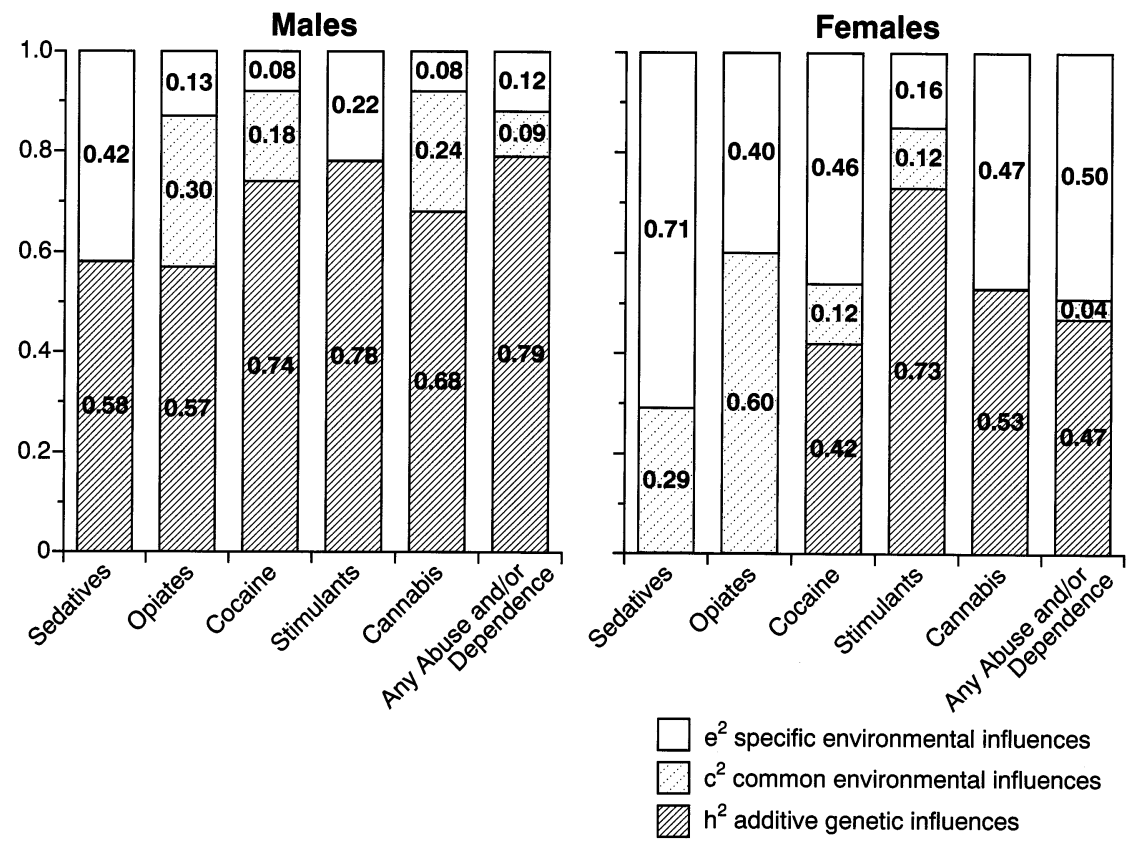

Fig. 2. Additive genetic, common environmental, and specific environmental influences on drug abuse and/or dependence for males and females.

largest interval, for sedative use in females, had lower and upper limits of 0.20 and 0.91 , respectively). For males, lower limits for confidence intervals around heritability estimates were zero for use of opiates, cocaine, cannabis, and any use and diagnoses of abuse/dependence of sedatives and opiates. Lower limit values greater than zero were obtained for use of sedatives and stimulants, dependence on stimulants, cannabis, and any drug, and abuse and/or dependence of cocaine, stimulants, cannabis, and any drug. For females, all lower limits of confidence intervals around heritability estimates were zero. The lower limit of confidence intervals for common environmental influences was zero for most measures among males, except cannabis and any drug use. Among females, lower limits larger than zero were found for opiate and cocaine use; and sedative and opiate abuse/dependence. Lower limits of confidence intervals around unique environmental estimates exceeded zero for all measures for both genders.

\section{Discussion}

The findings indicate genetic and environmental influences contribute both to illicit drug use and to the clinical diagnosis of illicit drug abuse/dependence. Evidence of both genetic and environmental influences was found for all drug classes for males and for most drug classes for females. With the exception of sedatives and opiates, heritability estimates were greater for drug abuse/dependence than for drug use, whereas environmental factors contributed more to drug use. Lower overall environmental influence on drug abuse/depen- dence was predominantly attributable to a reduction in common, rather than specific environmental factors. This suggests environmental influences shared by family members are more important to drug use than to becoming drug dependent (which is more genetically influenced). Possible environmental influences reported in the literature include location of residence (O'Malley et al., 1991; Warner et al., 1995), economic factors (Richman, 1977; Ambert, 1982; Smart and Murray, 1983), substance availability (Arnao, 1990; Newcomb and Felix-Ortiz, 1992; MacCoun and Reuter, 1997), peer groups (Kandel, 1985; Luthar et al., 1992), and family factors while growing up (Brook et al., 1983; Kandel, 1985; Newcomb and Bentler, 1988; Needle et al., 1990).

The relative influence of genetic and environmental influences varied somewhat by drug class. For cannabis use in males, for example, a relatively low heritability estimate $(0.17)$ was found along with a substantial common environmental influence (0.61). However, for cannabis abuse and/or dependence, genetic factors accounted for 0.68 of total variation in males, with an additional 0.24 attributable to common environmental influences. In contract, for sedative use in males, a heritability estimate of 0.74 was found, with negligible common environmental influences (0.02). For sedative abuse and/or dependence in males, 0.58 of variation was of genetic origin, with no evidence of common environmental factors. Differences in genetic and environmental influences across drug classes may reflect differences in drug abuse liabilities, actions on brain receptors, and/or psychological effects (Nestler and Aghajanian, 1997). 

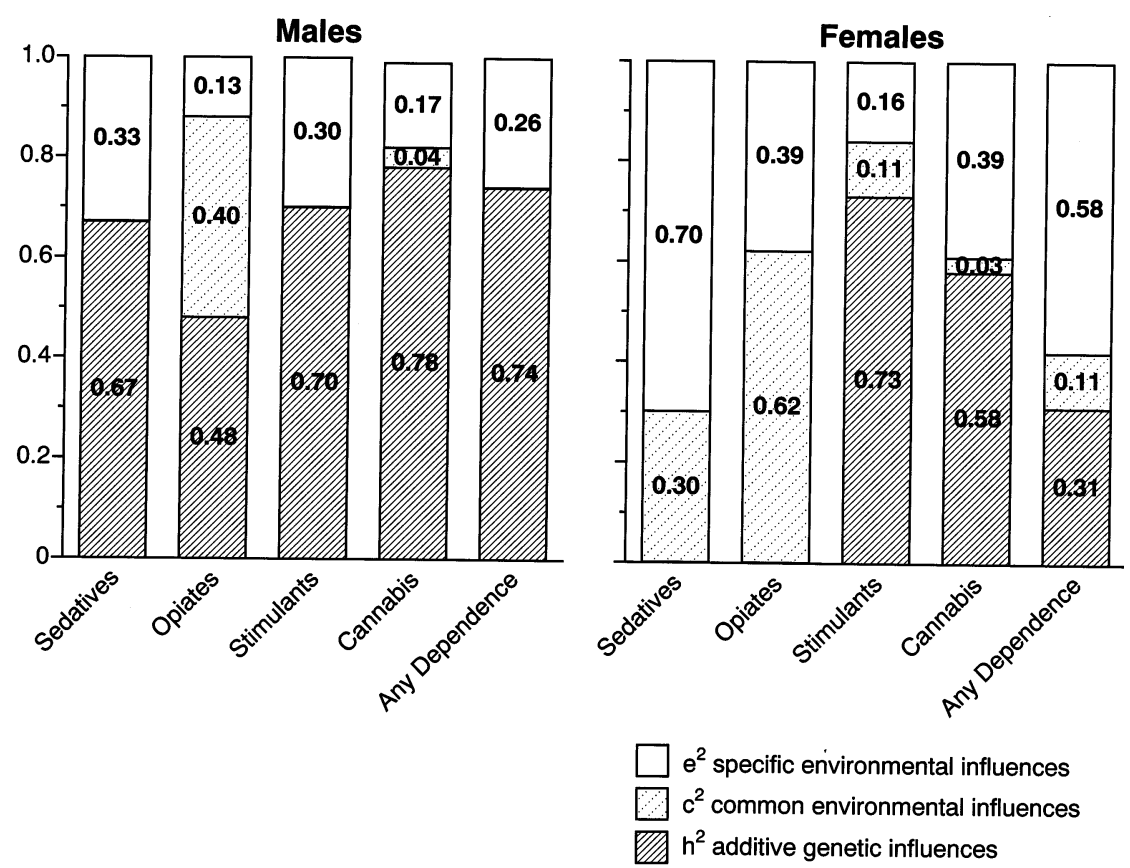

Fig. 3. Additive genetic, common environmental, and specific environmental influences on drug dependence for males and females.

\subsection{Relation to other studies}

In her study of Swedish twins, Pedersen (1981) reported a heritability of 0.28 for use of tranquilizers, and no evidence of genetic influences on sleeping pill use for both sexes combined. Inspection of MZ and DZ correlation coefficients presented separately for males and females, however, revealed the zero heritability for sleeping pill use was predominantly due to zygosity differences in males. Comparison of female correlations suggested a modest genetic component for sleeping pill use $(0.24)$. The present study reported heritability estimates of 0.74 and 0.18 for sedative use of males and females, respectively. Compared with results from the Pedersen study, there is agreement on heritability estimates for females, but there is considerable discrepancy between those for males. Discrepancy may be partly attributable to differences between the two studies in sample characteristics as well as measurement instruments. In addition, the Pedersen study was based on licit, rather than illicit drug use (N. Pedersen, personal communication).

Tsuang et al. (1996) reported heritability influences explained between 0.33 and 0.44 of variation in DSMIIIR abuse/dependence of marijuana, stimulants, sedatives, and opiates, with a heritability estimate of 0.34 for any abuse/dependence. In comparison, our heritability estimates for males were uniformly higher (range $0.57-0.79$ ), with genetic factors contributing 0.79 to any abuse/dependence. The Tsuang et al. (1996) study was based on a sample of male twins who both served in the military during the Vietnam Era, whereas our subjects were obtained from alcohol/drug treatment programs (Pickens et al., 1991). Several (Kaij, 1960; Goodwin et al., 1973; Gjone et al., 1996; Yates et al., 1996; van den Bree et al., 1997), though not all (Stevenson et al., 1992; Rende et al., 1993; Kendler et al., 1994) studies have indicated that increased severity of a condition is associated with greater genetic influences. Even though the origin of our sample was adjusted for in our structural equation models, greater severity of symptoms in treatment-based samples may have resulted in higher estimates of genetic influences.

Previously we reported lower heritability estimates for any substance abuse/dependence $(0.31$ for males and 0.22 for females) in a study that was based largely on the same subjects (Pickens et al., 1991). Statistical refinements in the present study are believed to account for the differences in study results. These include: (1) threshold for treatment status to account for the portion of the total population distribution the probands and their co-twins were taken from (2) specification of double ascertainment. Taking into account relative rarity of a sample will increase estimated genetic influences (Smith, 1970).

Findings in samples of adoptees and their biological and adoptive parents have also supported a role for genes in drug abuse. Cadoret and co-workers (Cadoret et al., 1986, 1995) have reported evidence of two genetic pathways to drug abuse: a direct path leading from alcohol abuse or dependence in the biological parent to drug abuse in the adoptee, and an indirect path from antisocial behavior in biologic relatives to antisocial behavior in adoptee to adoptee drug abuse. 


\subsection{Gender differences}

Heritability estimates in males generally exceeded those in females, while common environmental influences in females tended to be more important. In addition, specific environmental influences were greater in females than in males. Gender differences have been reported in factors moderating illicit drug use, as well as patterns of drug use (Kaplan and Johnson, 1992; Newcomb and Felix-Ortiz, 1992). Rates of use and problem use are typically higher in males than in females (Clayton et al., 1986; Warner et al., 1995; Powis et al., 1996).

In our treatment-based sample, this pattern was reversed with rates of use as well as abuse/dependence of any drug in female probands exceeding those in male probands. This suggests male probands in treatment may be more representative of the total male population, than female probands in treatment are of the total female population. In other words, affected males are closer to the mean of a normal distribution of liability to drug use and abuse/dependence for males, than are female probands on a liability distribution representing the female population. In contrast, we found frequency of use and abuse/dependence to be generally lower for female co-twins than for male co-twins, suggesting female co-twins are more representative of the general female population than their probands. Greater deviance of drug using and drug abusing/dependent female probands was also reflected by gender differences in thresholds. Estimated threshold values for females were higher than for males, indicating greater genetic and/or environmental influences were necessary for females to use and become dependent on illicit drugs. Differences in threshold values for males and females have also been implicated in other traits (i.e. antisocial behavior and crime (Cloninger et al., 1978)).

Findings from adoption studies also suggest gender differences in genetic influences on drug abuse. In a sample of male and female adoptees combined (Cadoret et al., 1986), and a sample of male adoptees only (Cadoret et al., 1995), evidence of a direct as well as a indirect genetic pathway to drug abuse was reported. However, in a sample of female adoptees only (Cadoret et al., 1996), support for only the indirect path was found, suggesting the possibility of gender-specific biological influences.

\subsection{Statistical considerations}

As is commonly recognized with moderate size twin samples (Neale and Miller, 1997), confidence intervals around heritability and environmental estimates in the present study were broad. They included zero for approximately half of the heritability estimates for males, and all heritability estimates for females. In addition, most confidence intervals around common environmental estimates also included zero for both genders. We used threshold modeling to account for prevalence of use and abuse/dependence in the general population. Thus low prevalence of use and abuse/dependence for certain drug types is expected in the twin sample, given a high threshold in the general population. However, the analyses should still be interpreted in light of the knowledge that relatively low cell counts will influence parameter estimates.

The present analyses were based on a full model including additive genetic, and common and specific environmental influences. For most analyses, both a non-zero additive genetic as well as a common environmental component was estimated. The combined effect of additive genetic influences and common environmental influences was significant for all measures of drug use and abuse/dependence, for both males and females. This indicated the importance of a familial component to drug use and drug abuse/dependence. However, because limited sample size reduced power (Neale et al., 1994) not all components reached significance. Although some of the $\mathrm{MZ} / \mathrm{DZ}$ correlation comparisons suggested possibility of non-additive genetic (D) influences, models including a $\mathrm{D}$, instead of $\mathrm{C}$ component never gave a significantly better fit to the data than more parsimonious models including only additive genetic and specific environmental factors.

We found heritability estimates for involvement with illicit drugs ranging from 0 to 0.78 . For males, they were significant $(P<0.05)$ for repeated use of sedatives and stimulants, cocaine abuse and/or dependence, and stimulant, cannabis, and any abuse/dependence. Common environmental influences were significant for use of cannabis and any drugs. For females, none of the heritability estimates reached significance, while common environmental influences were significant for opiate and cocaine use, and sedative and opiate abuse/dependence. Our sample size did not allow statistical comparisons of gender-specific genetic and environmental influences. Considerably larger samples would allow testing for gender differences in magnitudes of genetic and environmental influences.

It has been suggested that a finding of lower genetic influences for females than males might be (partly) attributable to differential effects of assortative mating for the sexes (Vanyukov et al., 1994). Therefore, it would be interesting to replicate our results in a sample including male and female twins as well as their parents. In addition, replication of this study in different racial/ethnic groups would also be informative. Because of the clinical nature of diagnoses used, a treatment rather than a population-based sample should be used to replicate the present findings. To date, however, we are not aware of any treatment sample with an adequate size to allow significant discrimination of herita- 
ble and environmental estimates of diagnostic systems of illicit drugs.

\subsection{Limitations}

The present results were based primarily on Caucasians in treatment for alcoholism and/or drug problems. How the results would generalize to nonCaucasians is unknown. While we would expect the results to generalize to other treatment populations, how they would generalize to other individuals with substance abuse who are not in treatment is unknown. Individuals in treatment for alcoholism are known to differ significantly from the general alcoholic population (Anthony and Helzer, 1991; Bucholz et al., 1994) Only $30.4 \%$ of subjects meeting criteria for drug abuse/dependence have talked to a doctor or other professional about their problems (Anthony and Helzer, 1991). Because of the nature of our sample, we chose a method of analysis which allowed statistical adjustment for treatment ascertainment.

An advantage of use of treatment samples in the study of illicit drug is that reasonable statistical power can be obtained with relatively few subject assessments. In the general population, relative low frequency of occurrence, in combination with illegality of involvement with illicit drugs makes it difficult to recruit sufficient sample sizes. Of all types of residences, prisoners and patients in residential treatment programs for alcohol and drug problems have been reported to be most likely to have a history of drug abuse/dependence syndromes (Anthony and Helzer, 1991). However, these groups of individuals are also the least likely to participate as volunteers in population-based twin studies. An additional advantage of our sample was that most were past the age of risk for drug use. In ECA data (Anthony and Helzer, 1991) the mean age of initiating illicit drug use was 17 , while mean age of first drug problem was 20 . In our sample, only 3 and $13 \%$ of analyzed twins were under the age of 17 and 20, respectively.

Increased understanding of the role of genes in combination with environmental influences in drug use, and clinical diagnosis of abuse/dependence will provide important clues to the etiology of drug problems. Our findings indicate the relative role of genes and the environment vary depending on substance considered, whether use, or abuse/dependence is the phenotype of interest, and by whether individuals are male or female. Molecular genetic efforts aimed at identifying genes in most types of illicit drugs might have increased chance of success when clinical diagnosis of abuse/dependence is used, rather than use. In addition, environmental factors may complicate molecular efforts more with females than with males.

\section{Acknowledgements}

This work was supported in part by the Hazelden Foundation, Center City, MN, and Public Health Services grants AA06500, DA05147, and AG06886.

\section{References}

Agresti, A., 1990. Categorical Data Analysis. Wiley, New York.

Akaike, H., 1987. Factor analysis and AIC. Psychometrika 52, 317332.

Ambert, A.M., 1982. Drug use in separated/divorced persons. Gender, parental status, and socio-economic status. Soc. Sci. Med. 16, 971-976.

American Psychiatric Association, 1980. Diagnostic and Statistical Manual of Mental Disorders, 3rd ed. American Psychiatric Association, Washington, DC.

Anthony, J.C., Helzer, J.E., 1991. Syndromes of drug abuse and dependence. In: Robins, L.N., Regier, D.A. (Eds.), Psychiatric Disorders in America, The Free Press, New York, pp. 116-154.

Arnao, G., 1990. A caveat: drug policy as factor of trends in trade and use of different substances. Int. J. Addict. 25, 1485-1488.

Boyd, J.H., Robins, L.N., Holzer, C.E.I., Von Korff, M., Jordan, B., Escobar, J.I., Kessler, L., 1985. Making diagnosis from DIS data. In: Eaton, W.W. (Ed.), Epidemiology Field Methods in Psychiatry: The NIMH Epidemiologic Catchment Area Program. Academic Press, Orlando, FL, pp. 209-231.

Brook, J.S., Whiteman, M., Gordon, A.S., 1983. Stages of drug use in adolescence: personality, peer, and family correlates. Dev. Psychol. 19, 269-277.

Bucholz, K.K., Helzer, J.E., Shayka, J.J., Lewis, C.E., 1994. Comparison of alcohol dependence in subjects from clinical, community, and family studies. Alcohol. Clin. Exp. Res. 18, 1091-1099.

Cadoret, R.J., Troughton, E., O'Gorman, T.W., Heywood, E., 1986. An adoption study of genetic and environmental factors in drug abuse. Arch. Gen. Psychiatr. 43, 1131-1136.

Cadoret, R.J., Yates, W.R., Troughton, E., Woodworth, G., Stewart, M.A., 1995. An adoption study demonstrating two genetic pathways to drug abuse. Arch. Gen. Psychiatr. 52, 42-52.

Cadoret, R.J., Yates, W.R., Troughton, E., Woodworth, G., Stewart, M.A., 1996. An adoption study of drug abuse/dependence in females. Comp. Psychiatr. 37, 88-94.

Caetano, R., Schafer, J., 1996. DSM-IV alcohol dependence and drug abuse/dependence in a treatment sample of whites, blacks and Mexican Americans. Drug Alcohol Depend. 43, 93-101.

Clayton, R.R., Voss, H.L., Robbins, C., Skinner, W.F., 1986. Gender differences in drug use: an epidemiological perspective. NIDA Res. Monogr. 65, 80-99.

Cloninger, C.R., Christiansen, K.O., Reich, T., Gottesman, I.I., 1978. Implications of sex differences in the prevalences of antisocial personality, alcoholism, and criminality for familial transmission. Arch. Gen. Psychiatr. 35, 941-951.

Eaton, W.W., Kessler, R.C., 1985. Epidemiological Field Methods in Psychiatry: The NIMH Epidemiological Catchment Area program. Academic Press, Orlando.

Gjone, H., Stevenson, J., Sundet, J.M., Eilertsen, D.E., 1996. Changes in heritability across increasing levels of behavior problems in young twins. Behav. Genet. 26, 419-426.

Goodwin, D.W., Schulsinger, F., Hermansen, L., Guze, S.B., Winokur, G., 1973. Alcohol problems in adoptees raised apart from alcoholic biological parents. Arch. Gen. Psychiatr. 28, 238243.

Grove, W.M., Eckert, E.D., Heston, L., Bouchard, T.J., Segal, N., Lykken, D.T., 1990. Heritability of substance abuse and antisocial 
behavior: a study of monozygotic twins reared apart. Biol. Psychiatr. 27, 1293-1304.

Gynther, L.M., Carey, G., Gottesman, I.I., Vogler, G.P., 1995. A twin study of non-alcohol substance abuse. Psychiatr. Res. 56, 213-220.

Heath, A.C., Neale, M.C., Hewitt, J.K., Eaves, L.J., Fulker, D.W., 1989. Testing structural equation models for twin data using LISREL. Behav. Genet. 19, 9-35.

Jang, K.L., Livesley, W.J., Vernon, P.A., 1995. Alcohol and drug problems: a mutivariate behavioural genetic analysis of co-morbidty. Addiction 90, 1213-1221.

Kaij, L., 1960. Alcoholism in twins: studies on the etiology and sequals of abuse of alcohol. In: Anonymous Alcoholism in Twins. Almqvist and Wiksell, Lund, pp. 17-38.

Kandel, D., 1985. On processes of peer influences in adolescent drug use: a developmental perspective. Adv. Alcohol Subst. Abuse 4, $139-163$.

Kaplan, H.B., Johnson, R.J., 1992. Relationships between circumstances surrounding initial drug use and escalation of drug use: moderating effects of gender and early adolescent experiences. In: Glantz, M.D., Pickens, R.W. (Eds.), Vulnerability to Drug Abuse. American Psychological Association, Washington, DC, pp. 299358.

Kendler, K.S., Neale, M.C., Heath, A.C., Kessler, R.C., Eaves, L.J., 1994. A twin-family study of alcoholism in women. Am. J. Psychiatr. 151, 707-715.

Koob, G.F., Nestler, E.J., 1997. The neurobiology of drug addiction. J. Neuropsychol. Clin. Neurosci. 9, 482-497.

Kosten, T.A., Gawin, F.H., Kosten, T.R., Rounsaville, B.J., 1993. Gender differences in cocaine use and treatment response. J. Subst. Abuse Treat. 10, 63-66.

Luthar, S., Anton, S., Merikangas, K., Rounsaville, B.J., 1992. Vulnerability to drug abuse among opioid addicts' siblings: individual, familial, and peer influences. Comp. Psychiatr. 33, 190196.

MacCoun, R., Reuter, P., 1997. Interpreting Dutch cannabis policy: reasoning by analogy in the legalization debate. Science 278 , $47-51$.

Morishima, H.O., Whittington, R.A., 1995. Species-, gender-, and pregnancy-related differences in the pharmacokinetics and pharmacodynamics of cocaine. NIDA Res. Monogr. 158, 2-21.

Neale, M.C., 1997. Mx: Statistical Modelling. Department of Psychiatry, Box 126 MCV, Richmond, VA 23298.

Neale, M.C., Cardon, L.R., 1992. Methodology for Genetic Studies of Twins and Families. Kluwer, Dordrecht.

Neale, M.C., Miller, M.B., 1997. The use of likelihood-based confidence intervals in genetic models. Behav. Genet. 27, 113-120.

Neale, M.C., Eaves, L.J., Kendler, K.S., 1994. The power of the classical twin study to resolve variation in threshold traits. Behav. Genet. 24, 239-258.

Needle, R.H., Su, S.S., Doherty, W.J., 1990. Divorce, remarriage, and adolescent substance use: a prospective longitudinal study. J. Marriage Fam. 52, 157-169.

Nestler, E.J., Aghajanian, G.K., 1997. Molecular and cellular basis of addiction. Science 278, 58-63.

Newcomb, M.D., Bentler, P.M., 1988. The impact of family context, deviant attitudes, and emotional distress on adolescent drug use: longitudinal latent variable analyses of mothers and their children. J. Res. Pers. 22, 154-176.
Newcomb, M.D., Felix-Ortiz, M., 1992. Multiple protective and risk factors for drug use and abuse: cross-sectional and prospective findings. J. Pers. Soc. Psychol. 63, 280-296.

O’Malley, P.M., Johnston, L.D., Bachman, J.G., 1991. Quantitative and qualitative changes in cocaine use among American high school seniors, college students, and young adults. NIDA Res. Monogr. 110, 19-43.

Pedersen, N., 1981. Twin similarity for usage of common drugs. Prog. Clin. Biol. Res. 69C, 53-59.

Pickens, R.W., Svikis, D.S., McGue, M., Lykken, D.T., Heston, L., Clayton, P.J., 1991. Heterogeneity in the inheritance of alcoholism: A study of male and female twins. Arch. Gen. Psychiatr. 48, 19-28.

Powis, B., Griffiths, P., Gossop, M., Strang, J., 1996. The differences between male and female drug users: community samples of heroin and cocaine users compared. Subst. Use Misuse 31, 529543.

Regier, D.A., Farmer, M.E., Rae, D.S., Locke, B.Z., Keith, S.J., Judd, L.L., Goodwin, F.K., 1990. Comorbidity of mental disorders with alcohol and other drug abuse. J. Am. Med. Assoc. 264, 2511-2518.

Reich, T., James, J.W., Morris, C.A., 1972. The use of multiple thresholds in determining the mode of transmission of semi-continuous traits. Ann. Hum. Genet. 36, 163-184.

Rende, R.D., Plomin, R., Reiss, D., Hetherington, E.M., 1993. Genetic and environmental influences on depressive symptomatology in adolescence: individual differences and extreme scores. J. Child Psychol. Psychiatr. Allied Discipl. 34, 1387-1398.

Richman, A., 1977. The epidemiology of drug abuse: current issues. Ecological studies of narcotic addiction. NIDA Res. Monogr. 1, 173-196.

Robins, L.N., Helzer, J.E., Croughan, J., Williams, J.B.W., Spitzer, R.L., 1981. The NIMH Diagnostic Interview Schedule: Version III. US Public Health Service publ. ADM-T

Smart, R.G., Murray, G.F., 1983. Drug abuse and affluence in five countries: a study of economic and health conditions, 1960-1975. Drug Alcohol Depend. 11, 297-307.

Smith, C., 1970. Heritability of liability and concordance in monozygous twins. Ann. Hum. Genet. 34, 85-91.

Stevenson, J., Batten, N., Cherner, M., 1992. Fears and fearfulness in children and adolescents: a genetic analysis of twin data. J. Child Psychol. Psychiatr. 33, 977-985.

Tsuang, M.T., Lyons, M.J., Eisen, S.A., Goldberg, J., True, W.R., Lin, N., Meyer, J.M., Toomey, R., Faraone, S.V., Eaves, L.J., 1996. Genetic influences on DSM-III-R drug abuse and dependence: a study of 3372 twin pairs. Am. J. Med. Genet. 67, 473-477.

van den Bree, M.B.M., Johnson, E.O., Neale, M.C., Svikis, D.S., McGue, M., Pickens, R.W., 1997. Genetic analysis of diagnostic systems of alcoholism in males. Biol. Psychiatr. 43, 139-145.

Vanyukov, M.M., Moss, H.B., Tarter, R.E., 1994. Assortment for the liability to substance abuse and personality traits. Ann. NY Acad. Sci. 708, 102-107.

Warner, L.A., Kessler, R.C., Hughes, M., Anthony, J.C., Nelson, C.B., 1995. Prevalence and correlates of drug use and dependence in the United States. Results from the National Comorbidity Survey. Arch. Gen. Psychiatr. 52, 219-229.

Yates, W.R., Cadoret, R.J., Troughton, E., Stewart, M.A., 1996. An adoption study of DSM-IIIR alcohol and drug dependence severity. Drug Alcohol Depend. 41, 9-15. 